\title{
Prevalence of Malassezia pachydermatis in dogs with suspected Malassezia dermatitis or otitis in Slovakia
}

\author{
Eva Čonková1, Edina Sesztáková2, L'ubomír Páleník², Peter Smrčo ${ }^{3}$, Ján Bílek² \\ ${ }^{1}$ Department of Pharmacology, ${ }^{2}$ Small Animal Clinic, ${ }^{3}$ Department of Epizootology and Preventive Veterinary \\ Medicine, University of Veterinary Medicine and Pharmacy, Košice, Slovak Republic
}

Received May 26, 2009

Accepted April 6, 2011

\begin{abstract}
The aim of the study was to evaluate the prevalence of yeast Malassezia pachydermatis in dogs from Slovakia in relation to different predisposition factors (sex, age, body localisation, hair type, and season). Samples of ear swabs (58) and dermal swabs (131) from 147 dogs with clinical symptoms of suspected yeast dermatitis and/or otitis, were examined between June 2005 to June 2007. Relatively higher prevalence of $M$. pachydermatis was found in samples taken from males $(45.2 \%)$ than in females $(35.2 \%)$, and in geriatric dogs $(63.6 \%)$ than in young $(42.5 \%)$ or adult $(38.5 \%)$ dogs. Malassezia pachydermatis was isolated more often from ear swabs $(44.8 \%)$ than from skin swabs $(38.9 \%)$. Prevalence of $M$. pachydermatis was significantly higher $(p<0.05)$ in samples from the trunk area $(60.3 \%)$ than in samples from other skin areas. Significantly higher prevalence was found in samples from long-haired (51.5\%) and short-haired (45.9\%) dogs compared to smooth-haired $(21.4 \%)$ dogs. The prevalence was relatively higher in the samples taken in autumn $(52.6 \%)$, than the other seasons: spring $(36.1 \%)$, summer $(27.3 \%)$, winter $(45.7 \%)$; those differences were not significant. Malassezia pachydermatis is one of the most frequent yeasts isolated in dogs. Knowledge of factors predisposing to development of infection is valuable attribute of the correct diagnostic approach and case management.
\end{abstract}

Malassezia infection; predisposing factors; diagnosis

Malassezia pachydermatis is commensal, lipophylic basidiomycetous yeast of ovoid or ellipsoidal shape with a thick wall, without production of pseudomycellium (Mason and Evans 1991). It is commonly found on the skin of dogs; around $50 \%$ of clinically healthy dogs are carriers of this yeast. As a potential pathogen, it is isolated from skin of the mouth region, chin, external ear canal, anal sacs and from vagina and rectum (Guillot and Bond 1999; Carloti 2001; Nardoni et al. 2007).

Predisposing factors for development of clinical signs of infection include excessive production of sebum and/or decreased quality of sebum (seborrhoea), accumulation of moisture, damage of epidermis and concurrent dermatoses, atopy, and bacterial skin infections (Patterson and Frank 2002). Malassezia infection could be secondary to other primary diseases: endocrinopathies (hypothyroidism, hyperadrenocorticism, diabetes mellitus), keratinisation disorders, immunologic dysfunctions and skin neoplasias (Mauldin 2002). Some environmental factors, e.g. high temperature and humidity can be the causes of proliferation of Malassezia pachydermatis (Carloti 1991).

An important clinical symptom of the infection is intensive pruritus and strong odour of decomposing fat. Clinical signs of early stages of infection are alopecia, localised or generalised erythema, erythematous papules and macules, crusts and scaly skin on the facial area, the trunk, in the perianal and interdigital areas and in skin folds. In chronic cases hyperpigmentation and other secondary lesions due to scratching and licking have been reported (Carloti 2001; Dorogi 2002).

Malasseziosis occurs in dogs of various breeds (West Highland White Terrier, English Setter, Cocker Spaniel, Shih Tzu, German Shepherd) at different ages and in both sexes (Plant et al. 1992; Bond et al. 1996).

Address for correspondence:

MVDr. Eva Čonková, PhD.

University of Veterinary Medicine and Pharmacy

Department of Pharmacy and Pharmacology

Komenského 73,041 81 Košice, Slovak Republic
Phone: +421915984766

Fax: +421 0552981011

E-mail: conkova@uvm.sk

http://www.vfu.cz/acta-vet/actavet.htm 
The aim of this study was to evaluate the prevalence of the yeast Malassezia pachydermatis in dogs and its correlation with some environmental factors (season) and some physiological factors.

\section{Materials and Methods}

\section{Sampling procedures}

One hundred eighty nine samples from 147 dogs were examined between June 2005 and June 2007. The swabs were taken from skin and/or external ear canal of dogs examined at the Small Animal Clinic of the University of Veterinary Medicine and Pharmacy in Košice, Slovak Republic. The criteria for including dogs into the study were inflammatory skin lesions (dermatitis), alopecia, seborrhoea (with accompanying specific sweetish odour) and/or otitis with the accumulation of dark brown thick cerumen inside the ear canal. Age, sex, breed and the presence of main clinical symptoms (pruritus, erythema, alopecia, hyper pigmentation, seborrhoea, sweetish odour and presence of dandruffs) of the animals were recorded.

The samples for cytological and mycological analysis were taken from skin with pathological lesions (131 samples) and from the external ear canal ( 58 samples). Each swab for cytology was rolled onto a clean glass slide and stained with Hemacolor (Merck, Darmstadt, Germany); stained slides were checked under microscope at $\times 40$ magnification. At least 10 fields were examined. Samples with more than 5 yeasts on average per one field were considered positive.

Samples for mycological culture were taken with sterile swab and placed into transport media for yeast and fungi (Fungi-Quick - Dispolab, Žilina, Slovak Republic). Swabs were processed immediately $(<2 \mathrm{~h}$ after sampling), when not possible (late evening time of sampling) they were stored in refrigerator $\left(4^{\circ} \mathrm{C}\right)$ and processed within $18 \mathrm{~h}$, otherwise the sample was excluded of the study.

Mycological diagnostic procedures

The following cultivation media were used: Sabouraud's dextrose agar with chloramphenicol (HiMedia Laboratories, Mumbai, India) and modified Dixon's agar (30 g malt extract, $15 \mathrm{~g}$ agar, $3 \mathrm{~g}$ peptone, $20 \mathrm{~g}$ desiccated ox-bile, $10 \mathrm{ml}$ Tween $40,2 \mathrm{ml}$ glycerol, $400 \mathrm{mg}$ cycloheximid, $50 \mathrm{mg}$ chloramphenicol), prepared according to Senczek et al. (1999).

Inoculated Petri dishes were incubated at 37 or $32{ }^{\circ} \mathrm{C}$ for up to 5 days and monitored daily. Identification of the species Malassezia pachydermatis was based on macroscopic and microscopic appearance of colonies and its ability to grow on the medium with no lipid supplementation.

Sample with a growth of more than 5 CFU (colony-forming units) per plate was considered positive. The results of mycological cultivation were compared with the cytological evaluation of each sample. The person evaluating the result of mycological cultivation was not aware of the results of cytological evaluation of the particular sample.

Evaluated factors of predisposition

The influence of different possible predisposing factors on prevalence of Malassezia pachydermatis was evaluated.

The chosen indicators were: sex (male, female), age of the animal (adolescent $<2$ years, adults $2-9$ years, and geriatric $>9$ years), breed according to hair type (long-haired breeds, short-haired breeds, smooth-haired and rough-haired breeds) and season when the animal was presented, i.e. spring (April-June), summer, (JulySeptember), autumn (October-December) and winter (January-March). The following breeds were ranked as long-haired: German Keeshond, Golden Retriever, English Cocker Spaniel, American Cocker Spaniel, Yorkshire Terrier, long-haired Dachshund, Newfoundland dog, West Highland White Terrier. Breeds ranked as short-haired were: German Shepherd, Giant Schnauzer, Labrador Retriever, Poodle, Bohemian Wire-Haired Pointing Griffon, Cavalier King Charles Spaniel, English Springer Spaniel. Breeds ranked as smooth haired were: Shar-pei, French Bulldog, Whippet, Boxer, Chihuahua, Mops. There were no samples collected from rough haired dogs.

Skin surface was (for sampling and evaluation purposes) divided into several anatomical areas: head, interdigital area, axillar, inguinal and trunk area. Samples of the lesions occurring on the side, flank and back of the animals were ranked as the trunk area.

Breeds of dogs presented with otitis were divided according to the shape of the pinna into two groups. Breeds with erected and semi-erected pinna (ear shell) were German Shepherd, Chihuahua, Keeshond, Shar-pei, Mops, Giant Schnauzer. Breeds with pendulous pinna (ear shell), to which group animals with problematic curving of the ear canal and/or with narrow ear canal were also included (English and American Cocker Spaniel, Poodle, Whippet, Newfoundland dog, French bulldog, West Highland White Terrier, Golden Retriever, Labrador Retriever).

Fisher's exact test (StatSoft, U.K.) and Chi-square test (GraphPadPrism) were used for the analysis of association of variables. A probability value of $p<0.05$ was considered significant.

Prevalence of $M$. pachydermatis was evaluated in relation to several indicators such as sex, age, location of skin lesions, type of pinna (pendulous versus erected ears), type of haircoat (long versus short versus smooth) and the season of the year. All of those variables were evaluated separately for each year of the study and then in summary (for all years together). 
Table 1. Prevalence of Malassezia pachydermatis in dogs in relation to different predisposition factors during years 2005-2007

\begin{tabular}{|c|c|c|c|c|}
\hline Evaluated factor & $\begin{array}{l}2005(\mathrm{n}=19) \\
\text { positive/n }(\%)\end{array}$ & $\begin{array}{l}2006(\mathrm{n}=84) \\
\text { positive/n }(\%)\end{array}$ & $\begin{array}{l}2007(\mathrm{n}=44) \\
\text { positive/n (\%) }\end{array}$ & $\begin{array}{c}\text { Total }=147 \\
\text { positive/n (\%) }\end{array}$ \\
\hline \multicolumn{5}{|l|}{$\overline{\operatorname{Sex}}$} \\
\hline Male & $7 / 15(46.7)$ & $27 / 56(48.3)$ & $8 / 22(36.4)$ & 42/93 (45.2) \\
\hline Female & $1 / 4(25.0)$ & $9 / 28(32.1)$ & $9 / 22(40.9)$ & 19/54 (35.2) \\
\hline \multicolumn{5}{|l|}{ Age } \\
\hline$<2$ & $2 / 5 \quad(40.0)$ & $11 / 24(45.8)$ & 4/11 (36.4) & $17 / 40(42.5)$ \\
\hline $2-9$ & 4/11 (36.4) & $22 / 55(40.0)$ & $11 / 30(36.7)$ & $37 / 96(38.5)$ \\
\hline$>9$ & $2 / 3 \quad(66.7)$ & $3 / 5(60.0)$ & $2 / 3 \quad(66.7)$ & 7/11 (63.6) \\
\hline \multicolumn{5}{|l|}{ Location } \\
\hline Skin & $4 / 18(22.2)$ & $34 / 69(49.3)$ & $13 / 44(29.5)$ & $51 / 131(38.9)$ \\
\hline Face & - & 3/11 (27.3) & 2/9 (22.2) & $5 / 20 \quad(25.0)$ \\
\hline Interdigital area & $1 / 4(25.0)$ & $1 / 3 \quad(33.3)$ & $2 / 7 \quad(28.6)$ & 4/14 (28.6) \\
\hline Axillary area & $1 / 3(33.3)$ & $2 / 9 \quad(22.2)$ & $0 / 5$ & 3/17 (17.6) \\
\hline Inguinal area & - & $1 / 8 \quad(12.5)$ & $0 / 9$ & $1 / 17 \quad(5.9)$ \\
\hline Trunk & 2/11 (18.2) & $27 / 38(71.0)^{*}$ & $9 / 14(64.3)^{*}$ & $38 / 63(60.3) *$ \\
\hline Ears & 4/11 (36.4) & $14 / 34(41.2)$ & $8 / 13(61.5)$ & $26 / 58(44.8)$ \\
\hline Erected & $1 / 4(25.0)$ & 6/17 (35.3) & $1 / 2 \quad(50.0)$ & $8 / 23(34.8)$ \\
\hline Pendulous & $3 / 7(42.9)$ & $8 / 17(47.1)$ & 7/11 (63.6) & $18 / 35(51.4)$ \\
\hline \multicolumn{5}{|l|}{ Hair type } \\
\hline Long haired & $4 / 8(50.0)$ & $20 / 32(62.5)^{*}$ & $11 / 21(52.4)$ & $35 / 68(51.5)^{*}$ \\
\hline Short haired & $3 / 7(42.9)$ & $10 / 19(52.6)^{*}$ & 4/11 (36.4) & $17 / 37(45.9)^{*}$ \\
\hline Smooth haired & $1 / 4(25.0)$ & $6 / 26(23.1)$ & $2 / 12(16.7)$ & 9/42 (21.4) \\
\hline \multicolumn{5}{|l|}{ Season } \\
\hline Spring & - & 9/19 (47.4) & $4 / 17(23.5)$ & $13 / 36$ \\
\hline Summer & $2 / 8 \quad(25.0)$ & 4/14 (28.6) & - & $6 / 22(27.3)$ \\
\hline Autumn & $6 / 11(54.5)$ & $4 / 8 \quad(50.0)$ & - & $10 / 19(52.6)$ \\
\hline Winter & - & $19 / 43(44.2)$ & $13 / 27(48.1)$ & $32 / 70(45.7)$ \\
\hline
\end{tabular}

$* p<0.05$

\section{Results}

Table 1 shows the prevalence of Malassezia pachydermatis in relation to evaluated predisposing factors during the period from June 2005 till June 2007. The prevalence of M. pachydermatis was higher in samples from males $(45.2 \%)$ compared to females $(35.2 \%)$, but the difference was not significant. Age and season did not show a significant influence on the prevalence of $M$. pachydermatis, although positive isolates were found more frequently in animals of geriatric age $(63.6 \%)$ than in the groups of younger animals (adolescent $-42.5 \%$, adult $-38.5 \%$ ) and also the prevalence was higher during autumn $(52.6 \%)$ compared to other seasons (spring - 36.1\%, summer $-27.3 \%$, winter $-45.7 \%$ ).

The prevalence of $M$. pachydermatis was highest in samples from the trunk area $(60.3 \%)$. Difference between the influence of this variable and the other body areas on the prevalence of yeast was significant $(p<0.05)$.

Concerning the samples of ear swabs, higher prevalence of yeast was found in dogs with pendulous ears $(51.4 \%)$ compared to $34.8 \%$ in breeds with erected ears, but the difference was not significant. Considering the type of haircoat, the prevalence of $M$. pachydermatis was significantly higher in both long-haired and short-haired dogs $(51.5 \%$ and $45.9 \%$ respectively), than in smooth haired dogs $(21.4 \%)$. 
Comparison of the results of cytological examination with mycological cultivation showed that all samples classified "positive" by cytological examination (26 of ear swabs and 46 skin swabs) were proven positive also by mycological culture. In case of the ear swabs there was no difference between cytological and mycological identification, whereas some of the mycologically positive skin samples failed to be detected by cytological examination, e.g. 5 out of $51(9.8 \%)$ culture positive skin swabs were cytologically classified as "negative".

Clinical signs accompanying $M$. pachydermatis infection in 51 dogs were: seborrhoea $(54.9 \%)$, alopecia $(49.0 \%)$, pruritus $(41.2 \%)$, sweet odour $(33.3 \%)$, erythema $(27.5 \%)$ and hyperpigmentation $(5.9 \%)$.

\section{Discussion}

Factors associated with proliferation of $M$. pachydermatis and its transformation from commensal to pathogenic microorganism are poorly understood, however, most likely they reflect the disturbances of physical, chemical or immunological mechanisms limiting microbiological colonisation of skin (Morris 1999; Matousek et al. 2003; Cafarchia et al. 2005). Season of the year and related differences in temperature and humidity are affecting the development of Malassezia infection.

Sex, breed and age of the animal are considered important physiological factors of predisposition to infection.

In terms of sex there were no significant differences in prevalence of Malassezia dermatitis (Nardoni et al. 2004). However, some authors reported higher predisposition of male dogs to otitis associated with Malassezia infection compared to female dogs (Chaudhary and Mirakhur 2002). The results of our study show generally higher prevalence of $M$. pachydermatis in male dogs $(45.2 \%)$ than in females $(35.2 \%)$. This fact could be explained by the assumption that androgens cause higher production of sebum, which is one of the predisposing factors for development of latent infection.

While Plant et al. (1992) found no correlation between Malassezia dermatitis and the age of animals, Jeong et al. (2005) recorded high (63\%) prevalence in dogs 1-3 years old. Kiss et al. (1997) reported high (63\%) prevalence of Malassezia yeasts in otitis of animals 2-5 years old. Contrarily, according to our study the risk group seems to be the group of geriatric animals, with $63.3 \%$ prevalence of Malassezia infection.

Many studies confirm natural presence of Malassezia in dogs with apparently healthy skin (Cafarchia et al. 2005; Nardoni et al. 2007). In quantitative studies, Malassezia were isolated from at least one place in 18 out of 19 dogs $(95 \%)$ in healthy animals. According to the same study the area of chin was colonised by the highest number of yeasts, and the axillar area by the lowest number (Kennis et al. 1996). Similarly, in our study, in dogs with skin lesions the prevalence of Malassezia yeast was relatively low in the axilar area $(17.6 \%)$.

Cafarchia et al. (2005) reported 100\% prevalence of yeast in dogs with skin lesions in the inguinal and interdigital areas; the yeasts were isolated from all dogs with lesions. In our study the prevalence of pathogen in inguinal and interdigital area was much lower (5.9\% and $28.6 \%$, respectively); we found the highest prevalence of Malassezia organisms $(60.3 \%)$ on different areas of trunk surface (sides, back).

Prevalence of Malassezia yeasts in dogs with clinical signs of otitis in this study was relatively low $(44.8 \%)$, considering that similar prevalence $(40 \%)$ was found in samples taken from dogs with apparently normal ear canal (Haj sik et al. 1985). In dogs with otitis, slightly higher prevalence (57.1\%) was reported by Cafarchia et al. (2005), whereas in the above mentioned study of Haj sik et al. (1985), the pathogen was isolated in $72 \%$ of animals with otitis. Long and narrow canal, pendulous ears and increased production of sebum in the canal of some breeds (spaniels) are considered to be important predisposing factors for infection (Carloti 1991; Masuda et al. 2000). We have found increased prevalence 
of $M$. pachydermatis in the group of dogs with pendulous ears, but the difference was not significant.

Prevalence of $M$. pachydermatis varies among the breeds. There are studies showing high sensitivity of certain breeds, like Basset Hound, Dachshund, Cocker Spaniel, West Highland White Terrier, Poodle and Australian Silky Terrier (Plant et al. 1992; Bond et al. 1996), in other studies, on contrary, majority of dogs with positive isolates were crossbreeds (Cafarchia et al. 2005). For the purpose of evaluation of the results of this study, the dog breeds were divided into groups according to hair type. Significantly higher prevalence was found in longhaired and short-haired breeds as compared to smooth haired dogs.

There are many clinical manifestations of Malassezia dermatitis. In this study, dermatitis caused by $M$. pachydermatis was usually manifested in the area of back and sides as seborrhoeic, alopetic lesions with severe pruritus and sweetish odour. Similar symptoms were described by many other authors (Charach 1997; Jeong et al. 2005).

Comparing the diagnostic value of cytological examination with mycological cultivation the specificity of cytological examination in our study was very high $(100 \%)$, with very good sensitivity $(90.2 \%)$, whereas Cafarchia et al. (2005) despite high specificity reports poor sensitivity of cytological examination. Those results suggest that the cut off point considered in our study $(5$ yeast per $\times 40$ magnification field compared to 10 yeast per field as used by Cafarchia et al. (2005) may be more effective in detecting suspected clinically significant infection, while still obtaining excellent specificity. The limitation of this statement may be the relatively low number of samples examined, although similar criteria for cytological evaluation where described elsewhere (Medleau and Hnilica 2001).

$M$. pachydermatis is the most frequent yeast isolated in dogs. Knowledge of factors predisposing to the development of infection may be a valuable attribute of an appropriate diagnostic approach. We may expect higher prevalence of Malassezia infection in male, older dogs with long hair, at the trunk area, particularly during the season with increased air humidity.

\section{Acknowledgements}

This work was supported by Science and Techology Assistance Agency under the contract No. APVT-20-024104.

\section{References}

Bond R, Fergusson EA, Curtis CF, Craig JM 1996: Factors associated with elevated cutaneous Malassezia pachydermatitis populations in dogs with pruritic skin disease. J Small Anim Pract 37: 103-107

Cafarchia C, Gallo S, Romito D, Capelli G, Chermette R, Guillot J, Otranto D 2005: Frequency, body distribution, and population size of Malassezia species in healthy dogs and in dogs with localized cutaneous lesions. J Vet Diag Invest 17: 316-322

Carloti DN 1991: Diagnosis and medical treatment of otitis externa in dogs and cats. J Small Anim Pract 32: 394-400

Carloti DN 2001: Malassezia dermatitis in the dog. WSAVA World Congress, Vancouver. Available at: http://www.vin.com./VINDBPub/SearchPB/Proceedings/ PRO5000/PR00097.htm.

Guillot J, Bond R 1999: Malassezia pachydermatis: a review. Med Mycol 37: 295-306

Charach M 1997: Malassezia dermatitis. Can Vet J 38: 311-314

Chaudhary M, Mirakhur KK 2002: Studies on occurence of canine otitis. Indian Vet J 79: 748-749

Dorogi J 2002: Pathological and clinical aspects of the diseases caused by Malassezia species. Acta Microbiol Imunol Hun 49: 363-370

Hajsig M, Tadic V, Lukman P 1985: Malassezia pachydermatis in dog - significance of its localtion. Vet Arch 55: $259-266$

Jeong A, Hoh W, Jeong H, Eom K, Lee K, OHT 2005: Efficacy of itraconazole in 18 cases of Malassezia dermatitis in dogs. J Vet Clin 22: 90-93

Kennis RA, Rosser EJ Jr, Olivier NB, Walker RW 1996: Quantity and distribution of Malassezia organisms on the skin of clinically normal dogs. J Am Vet Med Assoc 208: 1048-1051.

Kiss G, Radvanyi S, Szigeti G 1997: New combination for the therapy of canine otitis externa. I. Microbiology of otitis externa. J Small Anim Pract 38: 57-60

Mason KV, Evans AG 1991: Dermatitis associated with Malassezia pachydermatis in 11 dogs. J Am Anim Hosp Assoc 27: 13-20 
Masuda A, Sukegawa T, Mizumoto N, Tani H, Miyamoto T, Sasai K, Baba E 2000: Study of lipid in the ear canal in canine otitis externa with Malassezia pachydermatis. J Vet Med Sci 62: 1177-1182

Matousek JL, Campbell KL, Kakoma I, Solter PF, Schaeffer DJ 2003: Evaluation of the effect of pH on in vitro growth of Malassezia pachydermatis. Can J Vet Res 67: 56-59

Mauldin EA 2002: Malassezia dermatitis in the dog: A retrospective histopathological and immunopathological study of 86 cases (1990-95). Vet Dermatol 7: 191-202

Medleau L, Hnilica KA 2001: Malasseziasis (Malassezia dermatitis). In: Small animal dermatology. A color atlas and therapeutic guide. W.B. Sanders Company USA: 41-42.

Morris DO 1999: Malassezia dermatitis and otitis. Vet Clin North Am Small Anim Pract 29: 1303-1310

Nardoni S, Mancianti F, Corazza M, Rum A2004: Occurrence of Malassezia species in healthy and dermatologically diseased dogs. Mycopathologia 157: 383-388

Nardoni S, Dini M, Taccini F, Mancianti F 2007: Occurence, distribution and population size of Malassezia pachydermatis on skin and mucosae of atopic dogs. Vet Microbiol 122: 172-177

Patterson AP, Frank LA 2002: How to diagnose and treat Malassezia dermatitis in dogs. Vet Med 97: 612-622

Plant JD, Rosenkratz WS, Griffin CE 1992: Factors associated with a prevalence of high Malassezia pachydermatitis numbers on dog skin. J Am Vet Med Assoc 201: 879-882

Senczek D, Siesenop U, Böhm KH 1999: Characterization of Malassezia species by means of phenotypic characteristics and detection of electrophoretic karyotypes by pulsed-field gel electrophoresis (PFGE). Mycoses 42: 409-414 